\title{
Household consumption when the marriage is stable
}

\author{
Laurens Cherchye Thomas Demuynck Bram De Rock
}

Frederic Vermeulen

\section{Appendix A: A necessary and sufficient condition for rational- izability by a stable matching (for online publication)}

Proposition 2 presents revealed preference conditions for a data set $\mathcal{D}$ to be rationalizable in the sense of Definition 5. These conditions are necessary and sufficient for rationalizability, and provide the basis for the necessary conditions in Proposition 1 in the main text. Importantly, as also discussed in Section 3 of the main text, the following necessary and sufficient conditions are nonlinear in nature, which makes them substantially more difficult to operationalize than the necessary conditions in Proposition 1.

Proposition 2. The data set $D$ is rationalizable by a stable matching if and only if there exist

a. for each matched pair $m \in M$ and $\sigma(m) \in W$, individual quantities $q_{m, \sigma(m)}^{m}, q_{m, \sigma(m)}^{\sigma(m)} \in R_{+}^{n}$ that satisfy

$$
q_{m, \sigma(m)}^{m}+q_{m, \sigma(m)}^{\sigma(m)}=q_{m, \sigma(m)},
$$

which define a matching allocation $\left\{q_{m, \sigma(m)}^{m}, q_{m, \sigma(m)}^{\sigma(m)}, Q_{m, \sigma(m)}\right\}_{m \in M}$,

b. for each unmatched pair $m \in M$ and $w \in W$ (with $\sigma(m) \neq w$ ), individual quantities $q_{m, w}^{m}$, $q_{m, w}^{w} \in R_{+}^{n}$ and public quantities $Q_{m, w} \in R_{+}^{k}$ that satisfy

$$
p_{m, w}\left(q_{m, w}^{m}+q_{m, w}^{w}\right)+P_{m, w} Q_{m, w}=y_{m, w},
$$

c. for each male $m \in M$, private quantities $q_{m, \emptyset}^{m} \in R_{+}^{n}$, and public quantities $Q_{m, \emptyset} \in R_{+}^{k}$, that satisfy

$$
p_{m, \emptyset} q_{m, \emptyset}^{m}+P_{m, \emptyset} Q_{m, \emptyset}=y_{m, \emptyset},
$$

d. for each female $w \in W$, private quantities $q_{\emptyset, w}^{w} \in R_{+}^{n}$ and public quantities $Q_{\emptyset, w} \in R_{+}^{k}$ that satisfy

$$
p_{\emptyset, w} q_{\emptyset, w}^{w}+P_{\emptyset, w} Q_{\emptyset, w}=y_{\emptyset, w}
$$


e. for each pair $(m, w)(m \in M, w \in W)$, personalized prices $P_{m, w}^{m}, P_{m, w}^{w} \in R_{++}^{k}$ that satisfy

$$
P_{m, w}^{m}+P_{m, w}^{w}=P_{m, w}
$$

as well as strictly positive Afriat numbers $U_{m, w}^{m}, U_{m, \emptyset}^{m}, U_{m, w}^{w}, U_{\emptyset, w}^{w}$ and $\delta_{m, w}, \delta_{m, \emptyset}, \lambda_{m, w}$, $\lambda_{\emptyset, w}$ (for any $m \in M$ and $w \in W$ ) that simultaneously meet the following constraints:

i. Afriat inequalities for all males $m \in M$, i.e. (for any $w, w^{\prime} \in W$ )

$$
\begin{aligned}
& U_{m, w}^{m}-U_{m, w^{\prime}}^{m} \leq \delta_{m, w^{\prime}}\left(p_{m, w^{\prime}}\left(q_{m, w}^{m}-q_{m, w^{\prime}}^{m}\right)+P_{m, w^{\prime}}^{m}\left(Q_{m, w}-Q_{m, w^{\prime}}\right)\right) \\
& U_{m, w}^{m}-U_{m, \emptyset}^{m} \leq \delta_{m, \emptyset}\left(p_{m, \emptyset}\left(q_{m, w}^{m}-q_{m, \emptyset}^{m}\right)+P_{m, \emptyset}\left(Q_{m, w}-Q_{m, \emptyset}\right)\right) \\
& U_{m, \emptyset}^{m}-U_{m, w^{\prime}}^{m} \leq \delta_{m, w^{\prime}}\left(p_{m, w^{\prime}}\left(q_{m, \emptyset}^{m}-q_{m, w^{\prime}}^{m}\right)+P_{m, w^{\prime}}^{m}\left(Q_{m, \emptyset}-Q_{m, w^{\prime}}\right)\right)
\end{aligned}
$$

ii. Afriat inequalities for all females $w \in W$, i.e. (for any $m, m^{\prime} \in M$ )

$$
\begin{aligned}
& U_{m, w}^{w}-U_{m^{\prime}, w}^{w} \leq \lambda_{m^{\prime}, w}\left(p_{m^{\prime}, w}\left(q_{m, w}^{w}-q_{m^{\prime}, w}^{w}\right)+P_{m^{\prime}, w}^{w}\left(Q_{m, w}-Q_{m^{\prime}, w}\right)\right), \\
& U_{m, w}^{w}-U_{\emptyset, w}^{w} \leq \lambda_{\emptyset, w}\left(p_{\emptyset, w}\left(q_{m, w}^{w}-q_{\emptyset, w}^{w}\right)+P_{\emptyset, w}\left(Q_{m, w}-Q_{\emptyset, w}\right)\right) \\
& U_{\emptyset, w}^{w}-U_{m, w}^{w} \leq \lambda_{m, w}\left(p_{m, w}\left(q_{\emptyset, w}^{w}-q_{m, w}^{w}\right)+P_{m, w}^{w}\left(Q_{\emptyset, w}-Q_{m, w}\right)\right)
\end{aligned}
$$

iii. individual rationality restrictions for all males $m \in M$ and females $w \in W$, i.e.

$$
\begin{aligned}
U_{m, \sigma(m)}^{m} & \geq U_{m, \emptyset}^{m}, \\
U_{\sigma(w), w}^{w} & \geq U_{\emptyset, w}^{w},
\end{aligned}
$$

iv. no blocking pair restrictions for all males $m \in M$ and females $w \in W$, i.e.

$$
\begin{aligned}
U_{m, \sigma(m)}^{m} & \geq U_{m, w}^{m}, \\
U_{\sigma(w), w}^{w} & \geq U_{m, w}^{w} .
\end{aligned}
$$

Thus, a necessary and sufficient condition for a data set $\mathcal{D}$ to be rationalizable by a stable matching is that it simultaneously satisfies the conditions (a)-(e) and (i)-(iv). Interestingly, the different conditions can be given a specific interpretation. First, the adding up constraints in (a)-(d) specify feasibility restrictions on the unknown quantities. In particular, condition (a) pertains to individual quantities for matched pairs $(m, \sigma(m))$, condition (b) to individual quantities and public quantities for unmatched pairs $(m, w)$, condition (c) to private and public quantities of males $m$ when single and, finally, condition (d) to private and public quantities of females $w$ when single. 
Next, condition (e) defines a formally similar feasibility constraint on the personalized prices $P_{m, w}^{m}$ and $P_{m, w}^{w}$ (for any matched or unmatched pair). Intuitively, these personalized prices represent the willingness-to-pay of individual members for the public consumption. Because they must add up to the actual prices $P_{m, w}$, they can actually be interpreted as Lindahl prices that correspond to a Pareto optimal provision of public goods.

Proposition 2 requires the existence of feasible quantities and prices that simultaneously meet the rationalizability conditions (i)-(iv). These rationalizability conditions are defined in terms of so-called Afriat numbers (after Afriat, 1967). First, the Afriat numbers $U_{m, w}^{m}, U_{m, \emptyset}^{m}$ represent male $m$ 's utilities in alternative decision situations (respectively, in the pair $(m, w)$ and as a single). A directly similar interpretation applies to the Afriat numbers $U_{m, w}^{w}$ and $U_{\emptyset, w}^{w}$, which represent female $w$ 's utilities. Next, the Afriat numbers $\delta_{m, w}, \delta_{m, \emptyset}$ (for male $m$ ) and $\lambda_{m, w}, \lambda_{\emptyset, w}$ (for female $w$ ) can be interpreted as marginal utilities of individual expenditures (or Lagrange multipliers) in the respective decision scenarios (using, for a given pair $(m, w)$, the personalized prices $P_{m, w}^{m}$ and $P_{m, w}^{w}$ to allocate public good expenditures to the individuals $m$ and $w)$.

Then, the inequalities in conditions (i) and (ii) make sure that there exist (non-negative, continuous, strictly increasing and concave) utility functions $u^{m}$ and $u^{w}$ that explain the data. First, the inequalities ensure that, for all matched couples, these functions satisfy the Pareto efficiency criterion in Definition 1. ${ }^{1}$ Next, they also guarantee that the Afriat numbers $U_{m, \emptyset}^{m}$, $U_{\emptyset, w}^{w}$ solve the individual maximization problems (1) and (2), and that the numbers $U_{m, w}^{m}$ and $U_{m, w}^{w}$ solve the maximization problem (3) (so that $U_{m, w}^{m}=\psi_{m, w}\left(U_{m, w}^{w}\right)$ ), i.e. $U_{m, w}^{m}$ and $U_{m, w}^{w}$ represent utilities that are situated on the Pareto frontier of the couple $(m, w)$. Given this, the conditions (iii) and (iv) impose consistency with the individual rationality criterion in Definition 2 and the no blocking pairs criterion in Definition 3. Note that the latter criterion is expressed in an alternative form. More specifically, given that the function $\psi_{m, w}\left(\bar{u}^{w}\right)$ is continuous and strictly decreasing (by Lemma 1), it is easy to see that the no blocking pair condition in Definition 3 is equivalent to the requirement that, for any man $m$ and woman $w$, there must exist at least one combination of $U_{m, w}^{m}$ and $U_{m, w}^{w}$ such that

$$
\begin{gathered}
U_{m, w}^{m}=\psi_{m, w}\left(U_{m, w}^{w}\right), \\
U_{m, w}^{m} \leq u^{m}\left(q_{m, \sigma(m)}^{m}, Q_{m, \sigma(m)}\right) \text { and } U_{m, w}^{w} \leq u^{w}\left(q_{\sigma(w), w}^{w}, Q_{\sigma(w), w}\right) .
\end{gathered}
$$

\footnotetext{
${ }^{1}$ See in particular Cherchye, De Rock and Vermeulen (2011), who present a revealed preference characterization of Pareto efficient (or collectively rational) household consumption in a setting that is formally similar to ours. The Afriat inequalities in their Proposition 1 are contained in the constraints (i) and (ii) of Proposition 2.
} 


\section{Appendix B: Proofs (for online publication)}

\section{Preliminaries}

We begin by proving the following lemma.

Lemma 1 The function $\psi_{m, w}\left(\bar{u}^{w}\right)$ is strictly decreasing and continuous over the interval $\left[0, \bar{U}_{m, w}^{w}\right]$.

Proof. Consider two utility levels $\bar{u}^{w}, \bar{u}^{w \prime}$ with $\bar{U}_{m, w}^{w} \geq \bar{u}^{w}>\bar{u}^{w \prime} \geq 0$. Given that every solution to (3) with utility level $\bar{u}^{w}$ is also feasible with the utility level $\bar{u}^{w \prime}$ (by strict monotonicity of the utility functions), we have that $\psi_{m, w}\left(\bar{u}^{w \prime}\right) \geq \psi_{m, w}\left(\bar{u}^{w}\right)$.

Let $q_{m, w}^{w}$ be part of the optimal solution for utility level $\bar{u}^{w}$, then $q_{m, w}^{w}$ is strictly positive for at least one good. Indeed, otherwise we have $u^{w}\left(0, Q_{m, w}\right)=0$ by assumption, which contradicts the inequality $u^{w}\left(0, Q_{m, w}\right) \geq \bar{u}^{w}>\bar{u}^{w \prime} \geq 0$. Given the continuity and strict monotonicity of the utility function $u^{w}$, we can take a tiny bit of these private goods from $w$ and give them to $m$ in such a way that $w$ still receives utility level $\bar{u}^{w \prime}$ and the budget constraint is still satisfied. From this redistribution, we see that the utility level of $m$ strictly increases, which means that the optimal solution must also strictly increase. This shows that $\psi_{m, w}\left(\bar{u}^{w \prime}\right)>\psi_{m, w}\left(\bar{u}^{w}\right)$.

To show continuity, we consider

$$
\begin{gathered}
\xi_{m, w}\left(\bar{u}^{m}\right)=\max _{q_{m, w}^{m}, q_{m, w}^{w}, Q_{m, w}} u^{w}\left(q_{m, w}^{w}, Q_{m, w}\right) \\
\text { s.t. } p_{m, w}\left(q_{m, w}^{m}+q_{m, w}^{w}\right)+P_{m, w} Q_{m, w} \leq y_{m, w}, \\
u^{m}\left(q_{m, w}^{m}, Q_{m, w}\right) \geq \bar{u}^{m} .
\end{gathered}
$$

The functions $\xi_{m, w}$ and $\psi_{m, w}$ are each other's inverse. To see this, assume that $\bar{u}^{w}=\xi_{m, w}\left(\bar{u}^{m}\right)$ and let $\left(q_{m, w}^{m}, q_{m, w}^{w}, Q_{m, w}\right)$ be the solution to the woman's optimization problem given $\bar{u}^{m}$. Clearly, this bundle satisfies all restrictions for the man's optimization problem, so $\psi_{m, w}\left(\bar{u}^{w}\right) \geq$ $\bar{u}^{m}$.

We can prove $\psi_{m, w}\left(\bar{u}^{w}\right)=\bar{u}^{m}$ by contradiction. Assume that $\psi_{m, w}\left(\bar{u}^{w}\right)>\bar{u}^{m}$ and let $\left(q_{m, w}^{m}, q_{m, w}^{w}, Q_{m, w}\right)$ be the optimal solution to the man's optimization problem for $\bar{u}^{w}$. This allocation is also feasible for the woman's optimization problem given $\bar{u}^{m}$. Moreover, $q_{m, w}^{m}$ is strictly positive for at least one good. Then, consider reallocating a tiny bit of these private goods from $m$ and give them to $w$ in such a way that $m$ still receives utility level $\bar{u}^{m}$ and the budget constraint is still satisfied. This allows the woman to reach a utility level strictly above $\bar{u}^{w}$. Thus, we obtain that $\xi_{m, w}\left(\bar{u}^{m}\right)>\bar{u}^{w}$, which gives the wanted contradiction.

We conclude that the function $\xi_{m, w}$ is a strictly monotone (invertible) function from an interval to an interval. As such, it must be continuous. 
Given this lemma, we can apply a general result of Alkan and Gale (1990) to show existence of a stable matching allocation. ${ }^{2}$

Proposition 3. For any set of men $M$ and women $W$ with, for all $m \in M$ and $w \in W$, utility functions $u^{m}$ and $u^{w}$, and given the incomes $y_{m, w}, y_{m, \emptyset}, y_{\emptyset, w}$ and prices $p_{m, w}, p_{m, \emptyset}$,

$p_{\emptyset, w}, P_{m, w}, P_{m, \emptyset}, P_{\emptyset, w}$, there exists at least one matching $\sigma$ that defines a stable matching allocation.

\section{Proof of Proposition 1}

This result defines necessary conditions for rationalizability that are based on the necessary and sufficient conditions in Proposition 2, which is presented in Appendix A. First, conditions (a) and (e) in Proposition 2 define the constraints

$$
q_{m, \sigma(m)}^{m}+q_{m, \sigma(m)}^{w}=q_{m, \sigma(m)} \text { and } P_{m, w}^{m}+P_{m, w}^{w}=P_{m, w}
$$

Next, the individual rationality constraints (iii) together with the Afriat inequalities (i) (for male $m$ ) and (ii) (for female $w$ ) in Proposition 2 give

$$
\begin{aligned}
0 & \leq\left[p_{m, \emptyset}\left(q_{m, \sigma(m)}^{m}-q_{m, \emptyset}^{m}\right)+P_{m, \emptyset}\left(Q_{m, \sigma(m)}-Q_{m, \emptyset}\right)\right], \\
0 & \leq\left[p_{\emptyset, w}\left(q_{\sigma(w), w}^{w}-q_{\emptyset, w}^{w}\right)+P_{\emptyset, w}\left(Q_{\sigma(w), w}-Q_{\emptyset, w}\right)\right] .
\end{aligned}
$$

In turn, this obtains

$$
\begin{aligned}
& y_{m, \emptyset} \leq p_{m, \emptyset} q_{m, \sigma(m)}^{m}+P_{m, \emptyset} Q_{m, \sigma(m)}, \\
& y_{\emptyset, w} \leq p_{\emptyset, w} q_{\sigma(w), w}^{w}+P_{\emptyset, w} Q_{\sigma(w), w} .
\end{aligned}
$$

Similarly, the no blocking pairs condition (iv) together with the Afriat inequalities (i) and (ii) give that, for all $m, w$ such that $\sigma(m) \neq w$,

$$
\begin{aligned}
0 & \leq\left[p_{m, w}\left(q_{m, \sigma(m)}^{m}-q_{m, w}^{m}\right)+P_{m, w}^{m}\left(Q_{m, \sigma(m)}-Q_{m, w}\right)\right] \\
0 & \leq\left[p_{m, w}\left(q_{\sigma(m), w}^{w}-q_{m, w}^{w}\right)+P_{m, w}^{w}\left(Q_{\sigma(w), w}-Q_{m, w}\right)\right] .
\end{aligned}
$$

The first inequality states that the man $m$ should not prefer his allocation in $(m, w)$ over his matching allocation (in revealed preference terms). The second condition does the same for woman $w$. Now, adding these two equations together yields

$$
y_{m, w} \leq p_{m, w} q_{m, \sigma(m)}^{m}+p_{m, w} q_{\sigma(w), w}^{w}+P_{m, w}^{m} Q_{m, \sigma(m)}+P_{m, w}^{w} Q_{\sigma(w), w}
$$

\footnotetext{
${ }^{2}$ Because the result follows directly from Theorem 1 of Alkan and Gale (1990), we need not include a formal proof.
} 


\section{Proof of Proposition 2}

Necessity. As a first step to deriving our revealed preference characterization, we define the first order conditions that are used to formulate this characterization. In particular, we consider these conditions for the optimization models that underlie our criteria of individual rationality and no blocking pairs: ${ }^{3}$

1. We begin with the two optimization problems for individual rationality. First, we consider the problem

$$
\begin{aligned}
\left(q_{m, \emptyset}^{m}, Q_{m, \emptyset}\right)= & \underset{q^{m}, Q}{\arg \max _{0}} u^{m}\left(q^{m}, Q\right) \\
& \text { s.t. } p_{m, \emptyset} q^{m}+P_{m, \emptyset} Q \leq y_{m, \emptyset},
\end{aligned}
$$

i.e. $\left(q_{m, \emptyset}^{m}, Q_{m, \emptyset}\right)$ represents the optimal allocation for $m$ if he spends the income $y_{m, \emptyset}$. The first order conditions yield

$$
\begin{aligned}
\frac{\partial u^{m}\left(q_{m, \emptyset}^{m}, Q_{m, \emptyset}\right)}{\partial q^{m}} & \leq \delta_{m, \emptyset} p_{m, \emptyset}, \\
\frac{\partial u^{m}\left(q_{m, \emptyset}^{m}, Q_{m, \emptyset}\right)}{\partial Q} & \leq \delta_{m, \emptyset} P_{m, \emptyset},
\end{aligned}
$$

where $\delta_{m, \emptyset}$ is the Lagrange multiplier associated with the budget constraint and the expressions on the left hand side of the inequalities represent subdifferentials of the utility function $u^{m}$.

Similarly, for the problem

$$
\begin{aligned}
\left(q_{\emptyset, w}^{w}, Q_{\emptyset, w}\right)= & \arg \max _{q^{w}, Q} u^{w}\left(q^{w}, Q\right) \\
& \text { s.t. } p_{\emptyset, w} q^{w}+P_{\emptyset, w} Q \leq y_{\emptyset, w},
\end{aligned}
$$

we get the conditions

$$
\begin{aligned}
& \frac{\partial u^{w}\left(q_{\emptyset, w}^{w}, Q_{\emptyset, w}\right)}{\partial q^{w}} \leq \lambda_{\emptyset, w} p_{\emptyset, w}, \\
& \frac{\partial u^{w}\left(q_{\emptyset, w}^{w}, Q_{\emptyset, w}\right)}{\partial Q} \leq \lambda_{\emptyset, w} P_{\emptyset, w},
\end{aligned}
$$

where $\lambda_{\emptyset, w}$ is the Lagrange multiplier associated with the budget constraint.

\footnotetext{
${ }^{3}$ We remark the formal similarity between the Pareto efficiency criterion in Definition 1 and the no blocking pair condition in Definition 3. Essentially, the condition in Definition 3 reduces to the one in Definition 1 for $(m, w)$ with $w=\sigma(m)$. Therefore, we can follow a directly analogous reasoning as under item 2 . below to obtain the rationalizability conditions in Proposition 2 that pertain to our Pareto efficiency requirement (compare with Cherchye, De Rock and Vermeulen, 2011). For compactness, we do not include this reasoning here.
} 
2. Let us then turn to the optimization problems for no blocking pairs. Here, the optimization problem is defined as

$$
\begin{gathered}
\left(q_{m, w}^{m}, q_{m, w}^{w}, Q_{m, w}\right)=\arg \max _{q^{m}, Q} u^{m}\left(q^{m}, Q\right) \\
\text { s.t. } p_{m, w} q^{m}+P_{m, w} Q \leq y_{m, w}, \\
u^{w}\left(q^{w}, Q\right) \geq U_{m, w}^{w} .
\end{gathered}
$$

i.e. $\left(q_{m, w}^{m}, q_{m, w}^{w}, Q_{m, w}\right)$ represents the allocation chosen by $m$ if he could freely spend the entire income $y_{m, w}$ given that $w$ should receive utility level $U_{m, w}^{w}$. The corresponding first order conditions give

$$
\begin{aligned}
\frac{\partial u^{m}\left(q_{m, w}^{m}, Q_{m, w}\right)}{\partial q^{m}} & \leq \delta_{m, w} p_{m, w} \\
\mu_{m, w} \frac{\partial u^{w}\left(q_{m, w}^{w}, Q_{m, w}\right)}{\partial q^{w}} & \leq \delta_{m, w} p_{m, w} \\
\frac{\partial u^{m}\left(q_{m, w}^{m}, Q_{m, w}\right)}{\partial Q}+\mu_{m, w} \frac{\partial u^{w}\left(q_{m, w}^{m}, Q_{m, w}\right)}{\partial Q} & \leq \delta_{m, w} P_{m, w}
\end{aligned}
$$

where $\delta_{m, w}$ is the Lagrange multiplier associated with the budget constraint and $\mu_{m, w}$ is the Lagrange multiplier associated with the utility constraint.

In what follows, we use $\lambda_{m, w}=\delta_{m, w} / \mu_{m, w}, \frac{\partial u^{w}\left(q_{m, w}^{w}, Q_{m, w}\right)}{\partial Q}=\lambda_{m, w} P_{m, w}^{w}$ and $P_{m, w}^{m}=$ $P_{m, w}-P_{m, w}^{w}$ (which implies $\left.\frac{\partial u^{m}\left(q_{m, w}^{m}, Q_{m, w}\right)}{\partial Q} \leq \delta_{m, w} P_{m, w}^{m}\right)$.

In a final step, we can define the characterization in Proposition 2 by combining the above first order conditions with the postulated concavity property of the utility functions $u^{m}$ and $u^{w}$. In particular, concavity implies (for any $q^{m \prime}, q^{w \prime}, q^{m \prime \prime}, q^{w \prime \prime} \in \mathbb{R}_{+}^{n}$ and $Q^{\prime}, Q^{\prime \prime} \in \mathbb{R}_{+}^{k}$ )

$$
\begin{aligned}
u^{m}\left(q^{m \prime}, Q^{\prime}\right)-u^{m}\left(q^{m \prime \prime}, Q^{\prime \prime}\right) & \leq \frac{\partial u^{m}\left(q^{m \prime \prime}, Q^{\prime \prime}\right)}{\partial q^{m}}\left(q^{m \prime}-q^{m \prime \prime}\right)+\frac{\partial u^{m}\left(q^{m \prime \prime}, Q^{m \prime \prime}\right)}{\partial Q}\left(Q^{\prime}-Q^{\prime \prime}\right), \\
u^{w}\left(q^{w \prime}, Q^{\prime}\right)-u^{w}\left(q^{w \prime \prime}, Q^{\prime \prime}\right) & \leq \frac{\partial u^{w}\left(q^{w \prime \prime}, Q^{\prime \prime}\right)}{\partial q^{w}}\left(q^{w \prime}-q^{w \prime \prime}\right)+\frac{\partial u^{w}\left(q^{w \prime \prime}, Q^{w \prime \prime}\right)}{\partial Q}\left(Q^{\prime}-Q^{\prime \prime}\right) .
\end{aligned}
$$

Then, we obtain the rationalizability conditions in Proposition 2 by using $u^{m}\left(q_{m, w}^{m}, Q_{m, w}\right)=$ $U_{m, w}^{m}$, and $u^{w}\left(q_{m, w}^{w}, Q_{m, w}\right)=U_{m, w}^{w}(m \in M \cup\{\varnothing\}$ and $w \in W \cup\{\varnothing\})$.

Sufficiency. To obtain the sufficiency result, we consider

$$
\begin{aligned}
u^{m}\left(q^{m}, Q\right) & =\min _{w \in W \cup\{\varnothing\}}\left[U_{m, w}^{m}+\delta_{m, w}\left(p_{m, w}\left(q^{m}-q_{m, w}^{m}\right)+P_{m, w}^{m}\left(Q-Q_{m, w}\right)\right)\right], \\
u^{w}\left(q^{w}, Q\right) & =\min _{m \in M \cup\{\varnothing\}}\left[U_{m, w}^{w}+\lambda_{m, w}\left(p_{m, w}\left(q^{w}-q_{m, w}^{w}\right)+P_{m, w}^{w}\left(Q-Q_{m, w}\right)\right)\right] .
\end{aligned}
$$


Varian (1982) shows, in a unitary context, that $u^{m}\left(q_{m, w}^{m}, Q_{m, w}\right)=U_{m, w}^{m}$ and $u^{w}\left(q_{m, w}^{w}, Q_{m, w}\right)$ $=U_{m, w}^{w}(m \in M \cup\{\varnothing\}$ and $w \in W \cup\{\varnothing\})$. Using this, we can use a readily similar argument as in Varian (1982) (for the unitary consumption model) and Cherchye, De Rock and Vermeulen (2011) (for the collective consumption model) to show that the utility functions $u^{m}$ and $u^{w}$ defined above rationalize the data set $\mathcal{D}$ by a stable matching (i.e. the data solve the optimization problems underlying our stability criteria for these functions $u^{m}$ and $u^{w}$ ). 